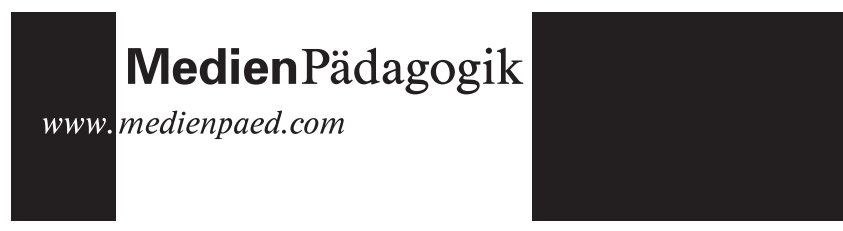

Heinz Moser

5.3.2001

\section{Medienpädagogische Forschung - Das Beispiel der Teletubbies}

Medienpädagogische Forschung hat in den letzten Jahren eine verstärkte Konjunktur erhalten. Diese verbindet sich dabei mit einer stärkeren Hinwendung zur qualitativen Forschung. Am Beispiel der Teletubbies versucht dieser Beitrag eine Zwischenbilanz zu ziehen und Stärken wie Schwächen dieser neuen Forschungslandschaft zu beleuchten.

Die vielen neuen Fernsehformate, die mit der zusätzlichen Konkurrenz privater Fernsehanbieter seit den Neunzigerjahren entwickelt wurden, sind auch zum Gegenstand des medienpädagogischen Diskurses geworden. Damit aber wurden die neuen Daily Soaps, Talkshows und Formate wie Big Brother auch Teil des Forschungsinteresses - was eine Vielzahl von Untersuchungen und wissenschaftlichen Diskussionen nach sich zog. Offensichtlich erhofft man sich von der Forschung Entscheidungshilfen in einem Feld, das sich im Gefolge technologischer Veränderungen und verstärkt marktorientierter Quotenorientierung der Angebote rasant entwickelt. Denn eine unvermittelt normative Medienpädagogik, die Kraft ihrer eigenen Autorität Medienereignisse bewertet, also Gefahren aufzeigt oder Entwarnung gibt, ist obsolet geworden. So erwartet man von der Wissenschaft eine Objektivierung subjektiver Meinungen und Haltungen und setzt auf eine empirische Untersuchung des Feldes.

Gleichzeitig ist auch im Umfeld der IKT-Technologien der Bedarf nach Forschung und Evaluation gestiegen. Denn die Einführung des Computers und des Internets in den Schulen ist weit weniger trivial als dies im ersten Moment erscheint. Rein technologische Ansätze, welche sich damit begnügen, den Schulen das Equipment zur Verfügung zu stellen, genügen nicht. Vielmehr ist pädagogisch-didaktische Entwicklungsarbeit notwendig
- und damit im Zusammenhang Forschung und sorgfältige Evaluation der jeweiligen Projekte (vgl. Weinreich, Schulz-Zander 2000).

Es ist denn auch bezeichnend für diese neue Medienforschung, dass sie sich nicht vornehm auf die abstrakten Grundlagenprobleme der Wissenschaft zurückzieht, sondern sich in das aktuelle Mediengeschehen mit ihrem analytischen Blick einmischt. So war die erste wissenschaftliche Studie zur Kultsendung Big Brother schon auf dem Markt, als die erste Staffel gerade mit der Erkürung des Siegers abgeschlossen wurde (vgl. Mikos 2000).

\section{Paradigmawechsel zu qualitativen Methoden}

Die Medienforschung der letzten Jahre ist aber nicht nur durch diese neue Aktualität geprägt, sie ist gleichzeitig durch einen paradigmatischen Wandel gekennzeichnet, wie er beispielshaft am Bereich der Wirkungsforschung aufgezeigt werden kann. Denn diese hat sich verstärkt in Abkehrung vom dominierenden rein empirisch-analytischen Forschungsansatz - qualitativ-empirischen Arbeitsformen zugewandt. Gerade aus systemtheoretischer Sicht bzw. aus der Perspektive der «cultural studies» ist hervorgehoben worden, dass die traditionelle Medienwirkungsforschung Bilder vom Zuschauer konstruiere, die jenseits der behaupteten Objektivität der Ergebnisse bereits Resultat eines Konstruktionsprozesses darstellten. Nach Winter (1995, S. 14) erscheine der Zuschauer wie das Kind, der Kriminelle oder der Patient in der Rolle des passiven, hilflosen und hirnlosen Konsumenten. Winter zieht daraus den Schluss: «Gerade die Erforschung der Medienaneignung legt es nahe, sich nicht von monolithischen Konzeptionen der Medien, der Wirklichkeit oder des Publikums leiten zu lassen, sondern die variable Interaktion von Zuschauer und Medien zu untersuchen, die ein aktiver Prozess der Bedeutungsproduktion ist. Erst in den kulturellen und sozialen Kontexten des Alltags gewinnen nämlich 〈Medienbotschaften〉 ihren Sinn» (Winter 1995, S. 15). Dadurch rückt die Medienforschung, ohne sich gleich in die Aporien einer zu distanzlos konzipierten Aktionsforschung zu begeben, in eine verstärkte Alltagsnähe. Sie ist gegenüber der empirisch-analytischen Forschung wie ihre Protagonisten betonen, näher bei der Praxis und dem Alltag, den sie zu erklären versucht (vgl. Flick 1995, S. 11).

Die Auswirkungen dieses Wandels sind in den letzten Jahren zunehmend greifbar geworden - typisch etwa im Wandel der Shell-Jugendstudien, die sich immer stärker am Muster qualitativer Forschung orientieren. Schon 
in der Ausgabe von 1992 hatte man sich entschlossen, neben eine Repräsentativbefragung auch Untersuchungsteile zu integrieren, in denen es um lebensgeschichtliche Rückerinnerung ging. Von den Autoren wurde dies damit begründet, dass sich mit solchen Retrospektiven auch im Rahmen von punktuellen Querschnittsbefragungen Hinweise auf lebensgeschichtliche Verläufe von Normalbiographien oder Belastungen durch kritische Lebensereignisse gewinnen liessen (vgl. Fischer/ Zinnecker 1992, S. 12). Sogar dort, wo die Zuschauerforschung sich noch stark an der traditionellen Umfrageforschung anlehnt, wird in der Ausschreibung von Forschungs- und Evaluationsprojekten immer häufiger verlangt, dass auch Methoden der qualitativen Forschung in Teilprojekte zu integrieren seien.

\section{Forschung als medienpädagogische Aufklärung}

Im vorliegenden Beitrag soll versucht werden, eine Zwischenbilanz dieser neuen - sich insbesondere auch auf qualitative Verfahren beziehenden medienpädagogischen Forschung zu ziehen. Dabei soll es indessen nicht allein um eine methodologische Auseinandersetzung gehen. Vielmehr wollen wir von konkreten Forschungsarbeiten ausgehen, indem wir uns auf einen exemplarischen Fall beziehen. Es handelt sich um eine Ausgabe der Zeitschrift TELEVIZION (2/1999) ${ }^{1}$, welches dem Thema «Teletubbies» gewidmet war. Gemäss Umschlagtext sollte dieses Heft «einen ersten Überblick über den internationalen Forschungsstand $\mathrm{zu}$ dieser Kinderserie» liefern. Die Teletubbies seien nicht nur bei Kindern weltweit ein Erfolg, sie würden von Erwachsenen in aller Welt thematisiert und diskutiert. Mit dieser Sendung werde die Diskussion um Kinder und

\footnotetext{
Nun könnte man gegen das hier gewählte Vorgehen einwenden, dass bei eine Kurzfassung von Forschungsprojekten notgedrungen Wesentliches weggelassen worden sei - und dies gerade bei den methodologischen Überlegungen. Unseres Erachtens ist dies allerdings keine Entschuldigung für Untrelassungen oder für die unpräzise Darstellung von Forschungsergebnissen. Denn schliesslich stützen sich die Leser/innen bei ihren Schlussfolgerungen meist ausschliesslich auf diese Berichte. Fehlen hingegen die Angaben wesentlicher Randbedingungen, ist eine zureichende Interpretation kaum mehr möglich. Zudem ist auch der Anspruch einer Berichterstattung über den «internationalen Forschungsstand» nur dann gerechtfertigt, wenn die dargestellten Arbeiten das Kriterium der «Transparenz» (vgl. Moser 1995, S. 118) erfüllen.
}

Fernsehen erneut angefacht sie seien Kultobjekt und Projektionsfläche für Kulturkritik und Homophobie.

Auch hier geht es offensichtlich darum, über Wissenschaft Ängste und Vorurteile abzubauen - oder wie es in der Einleitung zum dort publizierten Beitrag von Hake zur Rezeption in den skandinavischen Ländern heisst: «Forschungsergebnisse, die eine sachgerechte Beurteilung der Teletubbies ermöglichen, liegen in den skandinavischen Ländern noch nicht vor - sie werden allerdings dringend gebraucht» (Hake 1999, S. 14). Paul Löhr, Leiter des internationalen Zentralinstituts für das Jugend- und Bildungsfernsehen in München beschreibt die dahinterstehende Problematik im Editorial mit aller Deutlichkeit: «Dieses Programmformat ist neu. Das schreckt viele Erwachsene, weil Neues in allen Lebensbereichen zunächst ängstigt, und es geht eben um kleine Kinder, deren Schutzwürdigkeit ausser Frage steht» (Löhr 1999, S. 2). Gerade qualitative Forschung erscheint dagegen - so Hake - geeignet, «Erkenntnisse darüber zu gewinnen, wie Kinder dieses Programm erleben und welche Elemente für sie am faszinierendsten sind» (Hake 1999, S. 14).

Deutlich wird hier der veränderte Anspruch an die Forschung. Ging es früher oft darum, kritische Argumente gegen Gefahren der Medien empirisch zu untermauern (etwa die «fehlende Halbsekunde» bei Hertha Sturm), so soll heute mit Forschungsergebnissen eher kritischen Einwänden entgegengetreten werden. Medienforschung sieht sich damit oft in der Verteidigung gegen Kritik, die allzu sehr davon ausgeht, dass die Rezipienten lediglich Opfer von Reizen der Medienwelt sind. Demgegenüber wird im Rahmen qualitativer Studien aufgezeigt, wie Kinder und Jugendliche auch «aktive Nutzer» sind, die in der Interaktion mit Medienereignissen eigene Bedürfnisse und Intentionen zu realisieren versuchen.

Allerdings kann hier auch die Gefahr bestehen, einfach das Interpretationsmuster auszutauschen und kulturpessimistische Medienkritik affirmativ durch überschwängliche Zustimmung $\mathrm{zu}$ ersetzen. Etwas verwirrt meint denn auch ein journalistischer Kommentator zu diesem Wandel in der Neuen Zürcher Zeitung (NZZ): «Und en wegen ihrer Schmuddelthemen viel gescholtenen Talkshows wird inzwischen gar eine teilweise positive Wirkung bescheinigt. Sie könnten Orientierungshilfe leisten. Nun traut man gar den 〈Big Brother〉-Formaten zu, die Herzen bilden zu können» (Ausgabe vom 2. Februar 2001, S. 73). 


\section{Ein Fallbeispiel}

Doch wenden wir uns unserem Fallbeispiel zu: Die Rolle der blossen Objekte, bzw. der durch Reize überfluteten und damit überforderten Opfer thematisieren z.B. Sue Howard und Susan Roberts in der von ihren referierten australischen Studie zu den Teletubbies. Sie gehen darin von der Kritik aus, wonach sich Kinder in den ersten Lebensjahren noch zu wenig auf eine solche Fernsehsendung konzentrieren könnten. Mit ihrer Untersuchung versuchen sie eine wissenschaftliche Antwort auf die Medienberichterstattung in Australien, um kraft Autorität der Forschung und ihrer Daten dier Diskussion $\mathrm{zu}$ «versachlichen». Diese Intention kommt besonders deutlich im einleitenden Lead zum Ausdruck: «Viele Zeitungen in Australien haben die Teletubbies - wie in anderen Ländern auch - ohne jeglichen Beweis mit Homosexualität, Drogenkonsum u. $\ddot{\text { A. in }}$ Verbindung gebracht. Die ersten Ergebnisse einer Studie dagegen machen Hoffnung» (Howard/ Roberts 1999, S. 21)

Die Autorinnen zeichneten in dieser Studie vierzig Kinder unter zwei Jahren, welche sich die Teletubbies anschauten, auf Video auf, um gestützt auf empirisches Datenmaterial - solche Vorurteile zu widerlegen. Die Frage stellt sich jedoch, ihre Studie in der Lage ist, solche Erwartungen zu erfüllen. Dies bezieht sich insbesondere auf Fragen der methodologischen Qualitätskriterien qualitativer Forschung. Schreiben doch Howard/ Roberts gegen Schluss ihres Aufsatzes, dass ihre Studie noch in Arbeit sei, deshalb könnten die Resultate zu diesem Zeitpunkt noch nicht detailliert veröffentlicht werden., sowie an anderer Stelle: «Wir sind kurz davor, mit der detaillierten Analyse unserer Ergebnisse zu beginnen, daher können wir momentan nicht weiter darauf eingehen» (Howard/ Roberts 1999, S. 27).

Obwohl mit einer detaillierten Analyse der Ergebnisse noch nicht einmal begonnen wurde, werden dennoch weitreichende Folgerungen vorweggenommen. So heisst es im Text wörtlich: «Wir können jedoch mit Gewissheit sagen, dass die meisten Kinder, mit Ausnahme eines vierzehn Monate alten Babies, das nach fünf Minuten einschlief, beachtliche Zeitspannen lang genau aufpassten»(Howard/Roberts 1999, S. 27). Mit ihrer Untersuchung würden zwei «alte Weisheiten» in Frage gestellt: Die erste, wonach es nichts gebe, worauf sich Kleinkinder längere Zeit konzentrieren könnten, und die zweite, wonach das Gehirn der Kinder abschalte, wenn sie fernsehen. Offensichtlich hat die Aktualität der Sendung die Autorinnen dazu verführt, über etwas «mit Gewissheit» $5 / 17$
Aussagen zu machen und Folgerungen zu ziehen, worauf sie eigentlich, wie sie einige Zeilen später schreiben, «nicht weiter darauf eingehen» können.

Die Frage stellt sich, ob solche Schwächen eine Ausnahme darstellen. Konkret soll anhand der in TELEVIZION dargestellten Forschungsergebnisse die Frage gestellt werden, wie vertrauenswürdig die dort referierten Studien sind. Dies unter der Annahme, dass die Beiträge der Zeitschrift typisch für die Art und Weise sind, wie Arbeiten im Bereich der qualitativen Forschung im gegenwärtigen medienpädagogischen Diskurs erscheinen. Wenn wir also im Folgenden einige Praktiken bei der Veröffentlichung solcher Forschungsergebnisse kritisieren, dann gehen wir von einer doppelten Hypothese aus, nämlich dass diese nicht untypisch für die Art und Weise sind, wie mit qualitativem Datenmaterial umgegangen wird, bzw. dass die qualitative Medienforschung nicht immer mit dem notwendigen Methodenbewusstsein betrieben wird.

\section{Methodische Mängel}

Bereits eine erste Durchsicht der Beiträge von Heft 2/1999 der Zeitschrift TELEVIZION zeigt, dass viele der dort publizierten qualitativen Studien methodische Mängel aufweisen. So fehlen in den dargestellten Berichten häufig wesentliche Angaben über Grösse und Auswahl der Stichproben, verwendete Methoden, etc. Wenn an dieser Stelle auf einige konkrete Untersuchungen kurz eingegangen wird, so geht es zwar nicht um eine endgültige Beurteilung der jeweiligen Forschungsstudien. Es kann ja durchaus sein, dass solche Kriterien bei der Konzipierung der Forschungsarbeiten berücksichtigt wurden - und dass nur der publizierte Bericht darauf nicht eingeht. Dennoch betrachten wir dies als gravierendes Versäumnis. Denn wie soll der Leser einer solchen Arbeit daraus entnehmen können, für welche Gruppen und Personen die Daten gelten, wenn die Bedingungen, unter denen die Untersuchung stattfand, nur sehr lückenhaft wiedergegeben werden.

Roselyn Elliott beschreibt z.B. in ihrem Beitrag aus Australien eine Untersuchung mit Interviews in Kindertagesstätten, wobei es ihr darum geht, herauszuarbeiten, wie sich die dort Angestellten zu Fernsehprogrammen für Kleinkinder wie den Teletubbies verhalten. Befragt wurden dabei in rund 20-minütigen Interviews 20 bis 25 Personen aus solchen Einrichtungen, wobei deren Meinung in der Folge mit der 
Institution gleichgesetzt wird. Als Ergebnis werden prozentuale Werte angegeben, was dann zu Aussagen führt wie der nachfolgenden: «Es wurde nach den Gründen für bzw. wider eines TV- und Videoangebotes gefragt. Bei zwei Einrichtungen (9,5 Prozent) lag es daran, dass dafür keine Geräte vorhanden sind, zwei Einrichtungen (9,5 Prozent) gaben an, Fernsehen sei ihrer Meinung nach ein ungeeignetes Medium für Kinder in einem konventionellen Betreuung- und Erziehungsumfeld, drei Einrichtungen (14, 3 Prozent) gaben an, dass Kinder zu Hause sowieso schon zuviel fernsähen» (Elliott 1999, S. 38).

Obwohl diese Untersuchung gemäss ihrer Autorinnen zeigt, wie in vielen Betreuungsstätten eine sehr klare - negative - Einstellung gegenüber TVund Videoprogrammen zum Ausdruck kommt, fällt gleichzeitig auf, wie problematisch bei den geringen Anzahl von Befragten die Angabe von Prozentwerten ist. Auch wenn es sich lediglich um eine Vorstudie handelt, scheinen Ergebnisse äusserst problematisch, bei welchen eine einzelne Einrichtung bereits mit rund fünf Prozent zu Buche schlägt.

Schwierig einzuschätzen sind diese Ergebnisse aber auch, weil aus dem Text des Beitrags in der Zeitschrift TELEVIZION nicht hervorgeht, nach welchen Kriterien das Sampling - also die Auswahl der untersuchten Institutionen - stattfand. Für deutsche und schweizerische Leserinnen ist es mangels Kontextinformationen nicht abzuschätzen, um was für Institutionen es sich handelt - zum Beispiel was die im Text genannten «normalen Betreuungszeiten» bedeuten.

\section{Sampling und Verallgemeinerung im Rahmen qualitative Forschung}

Gerade diese Frage der Auswahl, welche mit darüber entscheidet, ob und wieweit die Ergebnisse von Untersuchungen sich generalisieren lassen, ist in qualitativen Studien oft ungelöst. Forscher und Forscherinnen beziehen sich auf Personen und Institutionen, zu welchen sie von ihrem Hintergrund her am einfachsten Zugang haben. Dabei wäre das Sampling, die Auswahl der zu untersuchenden Fälle, in der qualitativen Forschung besonders sorgfältig vorzunehmen, weil dazu nicht einfach die Regeln der Zufallswahl gelten, sondern inhaltliche Entscheidungen notwendig sind Eine Übersicht über mögliche Strategien des Sampling findet sich etwa bei Patton (1990, vgl. auch Moser 1995, S. 102 ff.). Probleme, die sich auf Unklarheiten im Sampling zurückführen lassen, zeigen zwei weitere der im selben Heft abgedruckten Studien:
-Lemish/Tidhar (1999, S. 42 ff.) berichten von ihrer israelischen Studie, in welcher sie 29 Personen interviewten. Zwanzig davon waren Mütter von 39 Kindern im Alter zwischen sechs Monaten und 10 1/2 Jahren; dazu wurden neun Experten befragt. Die Interviews seien von den Autorinnen durchgeführt worden und von diesen «beinahe wörtlich handschriftlich aufgezeichnet» (S. 43) worden. Auch wenn man über das «beinahe wörtlich» ${ }^{2}$ noch hinweg liest, kann man dies nicht mehr tun, wenn gleich anschliessend erklärt wird, dass alle Mütter aus der gebildeten Mittelschicht stammten und die Auswahl nach dem Schneeballprinzip ${ }^{3}$ erfolgt sei - ohne diese Methode indessen zu erläutern.

In der «Schlussbemerkung» des Aufsatzes heisst es dann zur Reichweite der Untersuchung: «Im grossen und ganzen zeigte unsere Studie, dass die Teletubbies in Israel bei Müttern der Mittelschicht und ihren Kindern sowie bei höher gebildeten Personen und bei Experten positiv aufgenommen werden. Der professionelle und edukative Charakter der Serie wird klar erkannt und geschätzt, und die Mütter erlauben ihren Kindern, die Sendung regelmässig anzusehen, manche ermutigen Sie sogar dazu» (Lemish, Tidhar 1999, S. 49).

Hier wird im Schnellschritt von den 29 Befragten generell auf «Mütter der Mittelschicht» sowie «höher gebildeten Personen und Experten» geschlossen. Zwar wird dies durch die Formulierung «im grossen und ganzen» wieder abgeschwächt, trotzdem scheinen mir die dargestellten Folgerungen aufgrund des Datenmaterials überzogen. Dass diese Untersuchung möglicherweise ein breiteres Verhalten in der Mittelschicht widerspiegelt, soll hier zwar nicht bestritten werden. Dennoch könnte nur aufgrund einer klar beschriebenen Strategie des Samplings plausibel gemacht werden, inwieweit eine gewisse Verallgemeinerung zulässig ist. Möglicherweise müssten auch weitere Forschungsverfahren (z. B. im Rahmen herkömmlich empirisch-analytischer Ansätze) triangulativ beigezogen werden, um so weitreichende Generalisierungen zureichend abzustützen. Mehr als einen «Einblick in diesem Themenbereich», wie es

Hier wären weitere Auusführungen zur Transkriptionsmethode angebracht. Der Ausdruck «beinahe wörtlich» ist eher dazu angetan Skepsis gegenüber der Datenqualität zu erzeugen

3 Das Schneeballprinzip ist oft nur ein anderer Ausdruck dafür, dass man in die Auswahl diejenigen einbezieht, die sich gerade anbieten - ohne weitere inhaltliche Selektionskriterien. Deshalb auch die hier geäusserte Skepsis. 
die Autorinnen bei der Einführung ihrer Studie versprechen, leis-tet diese denn letztlich auch nicht.

- Ähnlich unklar bleibt er es, wenn Höller/Müller (1999, S. 51ff.) ihre qualitative Untersuchungen von elf Kindern einer Kindertagesstätte im Alter von zwei bis fünf Jahren präsentieren. Es fehlen hier alle Angaben zur Frage der Auswahl (warum gerade diese Kindertagesstätte beziehungsweise gerade diese Kinder). Auch hier wird wiederum in fahrlässiger Weise generalisiert: Einmal werden im fetter Schrift zusammenfassende Aussagen als Überschriften gesetzt, welche die auf die befragte Gruppe bezogenen Textpassagen suggestiv zu allgemeingültigen Sätzen «verdichten»:

«Die Teletubbies sind für Schulkinder problemlos $\mathrm{zu}$ verarbeiten und bieten Anregungen zum Mitmachen» (S. 51).

«Vier- bis fünfjährige Kinder können sich während des Zuschauen entspannen, erhalten jedoch keine neuen Entwicklungsanreize» (S. 52).

Ähnlich generalisierend ist die Schlussfolgerungen der Autor/innen, wenn sie apodiktisch festhalten: «Zusammenfassend lässt sich sagen, dass keine negativen Einflüsse auf die Entwicklung der Kinder zu erwarten sind. Die Sendung entspricht den entwicklungsbedingten Bedürfnissen und Interessen von Vorschulkindern» (Höller/Müller 1999, S. 52). Solche weitreichenden Aussagen werden aufgrund von Interviews mit Eltern und Erzieherinnen formuliert, von denen man nicht einmal weiss, wie lange sie dauerten, sowie über Beobachtungen anhand von Videos, von denen nur berichtet wird, dass sie «auf dem Hintergrund alters- und entwicklungsspezifischer Merkmale ausgewertet wurden.» Weil zudem im Bericht nicht angegeben wird, wo der ausführliche Forschungsbericht zu beziehen ist, besteht auch keine Möglichkeit für die Leser/innen, sich ein genaueres Bild von der Forschungsarbeit zu machen.

Um diese Kritik nicht misszuverstehen: Es kann durchaus hilfreich sein, kleine Untersuchungen im genannten Sinne durchzuführen, um genauere Beobachtungen und Hinweise zur Auseinandersetzung von kleine Kindern mit den Teletubbies zu erhalten. Schwierig wird es jedoch, wenn eine solche Untersuchung im Kontext von Studien erscheint, welche gemäss Selbstverständnis der Herausgeber/innen einen Überblick über den internationalen Forschungsstand geben wollen.

Zusammenfassend scheint mir das Problem der Generalisierung in qualitativen Forschungsarbeiten häufig ein Stolperstein. Denn einerseits konzentriert man sich auf die Beschreibung von Einzelfällen, während die $9 / 17$
Rezipienten solcher Arbeiten Antworten auf allgemein formulierte Fragestellungen erwarten. Wie Flick (1995, S. 254) deutlich macht, liegt hier auch ein Dilemma: Denn einerseits liegt die Stärke qualitativer Forschung gerade darin, dass ihr Ansatzpunkt die auf spezifische Kontextbedingungen bezogene Analyse ist. Dadurch gewinnt sie ihre spezifische Aussagekraft und Bedeutung. Bei der Generalisierung dagegen wird dieser Kontextbezug wieder aufgegeben, um zu untersuchen, wieweit die gefundenen Zusammenhänge auch unabhängig von diesen Kontextbedingungen gelten.

Dabei ist die Übertragung von Ergebnissen zwischen Kontexten nur sehr vorsichtig vorzunehmen. Weder hilft es, die spezifischen Bedingungen $\mathrm{zu}$ übergehen, indem man gleich die Universalität der Geltung postuliert, noch kann es genügend, dass die Plausibilität der Ergebnisse eine allgemeine Gültigkeit der Ergebnisse nahe legt. Oft kann denn auch nur die inhaltliche Analyse der unterschiedlicher Kontextbedingungen etwas über die Zulässigkeit von Verallgemeinerungen aussagen. Und inbesondere ist schon bei der Auswahl von Fällen und den dabei einbezogenen Variationen - dem Sampling - auf den Gesichtspunkt einer möglichen Generalisierung zu achten.

\section{Probleme der Absicherung aus dem Datenmaterial}

Eine weitere qualitative Studie stellt Petra Best In Ihrem Beitrag «Die Teletubbies in der Elternberatung» (1999, S. 53) vor. Anhaltspunkte für die Frage, wie die Teletubbies bei jüngeren Kindern im Vorschulalter ankommen, sollte eine Kinderbefragung geben, die im Juli 1999 in mehreren Kindergruppen durchgeführt wurde. Zur Methode schreibt die Autorin: «Über die teilnehmende Beobachtung während der Rezeption einer Teletubbies-Folge sowie in anschliessenden Gesprächen und spielerischen Aktionen holten wir die Sichtweise und Urteile der Drei- und Vierjährigen ein»(1999, S. 53). Mehr erfährt man darüber nicht, wie auch die ganze Untersuchung auf einer einzigen Seite dargestellt ist. Aufgrund undurchsichtiger Kriterien zitiert die Autorin einzelne Aussagen und referiert Verhaltensmuster von Vorschulkindern. Hauptergebnis ist offensichtlich ein bereits im Lead angetexteter Sachverhalt: «Für die Beantwortung von Warum-Fragen scheinen die Teletubbies wenig geeignet (Best 1999, S. 53). Plausibel begründet wird dies mit dem Datenmaterial allerdings nicht; es wäre wohl auf dem Raum einer einzigen Seite auch nicht zu leisten. Wenn es in diesem Beitrag als abschliessende Vermutung 
hinzugezogen, wobei die Autorin bei der Angabe von absoluten Zahlen bleibt; und sie betont explizit, dass Verallgemeinerungen nur sehr vorsichtig zu ziehen seien (Götz 1991, S. 58). Problematisch bleiben die Schlussfolgerungen nur an wenigen Stellen; etwa wenn die Autorin schreibt: «Angesichts dieser Vielfalt von Aktivitäten scheint die Sendung harmlos, wenn nicht sogar kompetenzfördernd»(Götz, S. 58). Hier wird eine Bewertung gesetzt, die meines Erachtens aus dem Text nicht so klar hervorgeht. Vielmehr scheint die Tatsachenaussage moralisch aufgeladen. Was «harmlos» erscheint, ist eben pädagogisch gesehen auch «gut», oder wie es dann am Schluss des Aufsatzes heisst: «Die Teletubbies in diesem Geflecht als gut oder schlecht beurteilen zu wollen, heisst immer auch «den Zahn der Zeit〉 zu bewerten.

\section{Forschung, Vorurteile, Wertfreiheit}

Qualitative Forschung läuft schon deshalb, weil sie sinnhaften Erfahrungen nähersteht wie die empirisch analytische Forschung, schnell in Gefahr, ihre Erfahrungen auch «wissenschaftlich» zu bewerten, wobei dies oft so selbstverständlich erfolgt, wie wenn es sich um einen naturalistischen Schluss handelte. So scheinen auch die in TELEVIZION abgedruckten Untersuchungen zu den Teletubbies zu bestätigen, was die angesprochenen Rezipienten einer medienpädagogischen Fachzeitschrift ohnehin vermuten: Eine solche Fernsehserie ist für die Kleinkinder weit weniger gefährlich als dies oft in der öffentlichen Diskussion erscheint mag. Und der Schluss mag dann nicht mehr weit liegen, Eltern zu empfehlen, ihren Kinder dieses Produkt einer geglückten Innovation auch gleich empfehlen.

Nun hat allerdings die Diskussion um methodologische Fragen der Forschung schon in den 70er Jahren des letzten Jahrhunderts das Postulat der Wertfreiheit als Mythos entlarvt. Dennoch darf dies nicht bedeuten, dass hypothetische Annahmen der Forschung an der Realität nicht mehr scheitern können, weil alle Forschenden ohnehin nur ihre Vorurteile bestätigen wollen. Dies bedeutet aber, dass - gerade in der qualitative Forschung - ein distanzierender Rahmen notwendig ist, wie er durch die Systematisierung und an Regeln gebundene Auswertung von Daten möglich wird. Wo z.B. das Sampling an definierte Kriterien gebunden ist, wird die Gefahr verringert, dass nur Fälle in die Auswahl gelangen, welche die eigenen Urteile und Vorurteile bestätigen. ren/Bemerken/Kommentieren» geleitet, denen dann wiederum einzeln Ausschnitte und Szenen zugeordnet werden. Wo es notwendig ist, werden quantitative Zahlen (etwa im Rahmen von zwei Internet-Umfragen) $11 / 17$ 
In diesem Zusammenhang wäre auch an das Konzept der «grounded theory» zu erinnern (vgl. Glaser/Strauss 1967), das für sich beansprucht, aus dem Datenmaterial Theorien erst zu entwickeln, wobei der Forscher um ein «forcing» von bestimmten Vorurteilen zu vermeiden - im Entwicklungsprozess seine eigenen Deutungen möglichst zurückhalten muss. Man kann daran den Induktivismus kritisieren - wie wenn Forschende ihre eigenen theoretischen und lebensweltlichen Bezüge einfach ausschalten könnten. Dennoch scheint es als methodisches Prinzip ratsam, sich selbst als Faktor des Interpretationsprozesses in Rechnung zu setzen und sorgfältig zu kontrollieren-

Gleichzeitig wäre - gerade Akteuren im Medienbereich muss dies wohl nicht weiter ausgeführt werden - bei der Präsentation von Forschungsarbeiten auf die alte journalistische Regel der Trennung von Darstellung und Kommentar/ Empfehlungen zu verweisen. Überall dort, wo es um die Evaluation von Projekten oder um die Beschreibung umstrittener Medienereignisse (wie im Fall der Teletubbies) geht, wäre dies auf jeden Fall empfehlenswert. Denn Fakten können oft - je nach Standpunkt - ganz unterschiedlich interpretiert werden. Bei der Präsentation von Forschungsergebnissen wäre es deshalb wichtig, durch eine differenzierte Darstellung auch abweichende Interpretationen zuzulassen.

\section{Versuch eines Fazits}

Das hier bist dargestellte Beispiel der Teletubbies ist meines Erachtens in verschiedener Hinsicht typisch für die gegenwärtige Medienforschung. Die Art der publizierten Untersuchungen weist darauf hin, wie stark sich in den letzten Jahren Konzepte einer qualitativen Untersuchung verbreitet haben. Es scheint offensichtlich keiner Diskussion mehr zu bedürfen, dass Forschung sich heute an qualitativen Studien orientiert, die mit einer beschränkten Anzahl von Fällen operieren und eher in die Tiefe als in die Breite gehen. Dagegen mussten noch Theunert u.a. 1992 in ihrer Untersuchung «Zwischen Vergnügen und Angst - Fernsehen im Alltag von Kindern» sich für ihren qualitativ orientierten Ansatz methodisch explizit rechtfertigen. Sie schreiben in einem eigenen methodologischen Kapitel ihres Buches: «Aber die meisten Menschen glauben an die grosse Zahl. Umgeben von Statistiken können sie sich nur das als exakte Wissenschaft vorstellen, was sich in Prozenträngen ausdrücken lässt. Auch wir standen der Argumentation gegenüber, dass man mit einigen wenigen
Kindern doch keine allgemeinen Aussagen über Medien und ihre Verarbeitung machen kann...»(Theunert u.a. 1992, S. 210).

Gegenüber der früher klar im Vordergrund stehenden empirischanalytische Forschung werden qualitative Studien heute grundsätzlich als gleichwertig achtet. Dies mag nicht zuletzt damit zusammenhängen, dass sich in den letzten Jahren in diesem Bereich ein klares methodologisches Forschungsinstrumentarium entwickelt, das zum Teil auch in Hand- und Lehrbüchern kodifiziert ist (vgl. z.B. Lamnek 1993, Denzin/Lincoln 1994, Flick 1995,). Dieses Handwerkszeug reicht hin bis zu computergestützten Auswertungsverfahren (ATLASti, NUD.IST, Winmax etc.) welche die Kodifizierung und Interpretation der Daten erleichtern.

Allerdings ist die Orientierung solcher Verfahren an eindeutigen Regeln nicht so eng wie bei der Auswertung quantitativer Projekte, die sich an klar determinierten statistischen Auswertungsprozeduren ausrichten. In der qualitativen Forschung ist deshalb die Entwicklung eines Forschungsdesigns selbst schon ein Prozess, der interpretativ erfolgt - indem immer wieder Entscheidungen zu treffen sind, die inhaltlich zu begründen sind (etwa bei der Entwicklung von Codes zur Auswertung von Textmaterial). Dabei arbeiten die Forschenden im «hermeneutischen Zirkel», indem die aktuellen Interpretamente wieder den Sinn des Ganzen verändern können. Wo es indessen schnell gehen soll, weil ein aktuelles Medienereignis erklärt werden soll, besteht die Gefahr, dass man diesen komplexen Prozess einer Forschung, die sich über Schleifenbildung immer wieder selbstreflexiv auf sich selbst bezieht, zu verkürzen sucht, um einzelne plausible Erfahrungen als «Forschungsresultat» wiederzugeben. So mag es verführerisch sein, wenn z.B. ein umstrittenes Fernsehformat in den Medien diskutiert wird, sich schnell eine Schulklasse oder eine Kindergruppe zu «besorgen», um über wenige Interviews eine «wissenschaftliche Interpretation» zu liefern. Seriös scheint mir dies allerdings nicht. Vielmehr liegt die Gefahr auf der Hand, dass in Konsequenz eines solchen Vorgehens die Medienforschung leicht zum Spielball medienpolitischer Interessen werden könnte. Schliesslich ist es dann kein Problem mehr, für eine vorgefasste Meinung auch eine Untersuchung $\mathrm{zu}$ «organisieren», welche diese bestätigt.

Wenn hier auf die Notwendigkeit klarer Regeln für die qualitative Forschung Bezug genommen wird, so auch deshalb, weil sich die eben erreichten methodologischen Standards der qualitative Forschung gegenwärtig zum Teil beängstigend abschwächen. Vor allem in den USA geht 
Aus diesem Grund scheint es mir wichtig, dass eine qualitative orientierte Medienforschung ihre wachsende Reputation nicht wieder dadurch verliert, dass sie sich zu wenig um gültige Forschungsstandards kümmert. Wenn es auch notwendig war, dass gegenüber den Standard der empirisch-analytischen Forschung eine Öffnung auf neue Erfahrungsdimensionen erfolgte, so darf dies nicht bedeuten, dass im Sinne eines «anything goes» alles möglich wird. Gegenüber der hochsubjektiv angelegten traditionellen Hermeneutik, bei welche es letztlich nur der Interpret selbst war, dessen «Kunst der Interpretation» (Staiger) die Gültigkeit der Interpretation verbürgte, scheint es mir ein Fortschritt zu sein, dass die qualitative Forschung aus der Kunst stärker eine regelgeleitete Methodologie entwickelt hat. Aus dieser Perspektive erschiene es fatal, den erreichten Stand wieder aufzugeben.

Gleichzeitig scheint es mir auch notwendig, Forschungsarbeiten vermehrt auf die darin realisierten methodologischen Prinzipien zu hinterfragen. Denn die Qualität medienpädagogischer Forschung ist nur dann zu gewährleisten, wenn immer auch die methodischen Standards, denen solche Arbeiten genügen müssen, mitreflektiert werden. Eine solche kritische Diskussionstradition besteht indessen im deutschsprachigen Raum noch zu wenig. Im Vordergrund stehen meist die Inhalte und Themen, während man wenig Zeit auf eine ausführliche Methodendiskussion verwendet. So findet man auch in renommierten Publikationen immer wieder einzelne Beiträge, die kaum geeignet sind, das Vertrauen in qualitative Forschungsverfahren zu erhöhen. Dabei sollte es eigentlich keine Frage sein, dass Thesen, die aufgrund fragwürdiger Methodenarrangements zustande kommen, $\mathrm{zu}$ einer fruchtbaren Theoriebildung wenig beizutragen vermögen. Aus diesem Grund scheint es mir ausserordentlich wichtig, dass die Diskussion methodologischer Standards noch stärker zum Handwerk des medienpädagogischen Diskurses gehören muss. Dies war nicht zuletzt die Absicht, welche mit der Konzipierung des vorliegenden Heftes der Zeitschrift MedienPädagogik verbunden war.

\section{Literatur}

Best Petra, Die Teletubbies in der Elternberatung, in: TELEVIZION 2, 1999, S. $53 \mathrm{ff}$.

Denzin Norman K., Lincoln Yvonna S., Handbok of Qualitative Research, Thousand Oaks 1994 
Elliott, Rosalyn, Fernsehen in Kindertagesstätten, in: TELEVIZION 2, 1999, S. $38 \mathrm{ff}$.

Ellis Carolyn, Creating Criteria: An Ethnographic Short Story, in: Qualitative Inquiry 2000, S. 273 ff.

Fischer Arthur, Zinnecker Jürgen, Jugend 92. Band 1. Gesamtdarstellung und biographische Porträts, Opladen 1992

Flick Uwe, Qualitative Forschung, Reinbek 1995

Hake Karin, Nicht ohne Forschung, in: TELEVIZION 2, 1999, S. 14 ff.

Glaser Barney, Strauss Anselm, The Discovery of Grounded Theory, London 1967

Götz Maya, Begeisterung bei den Kindern - Besorgnis bei den Eltern, in: TELEVIZION 2, 1999, S. 54 ff.

Höller Claudia, Müller Sabine, «Ah oh - jetzt ist Teletubby-Zeit.» Ergebnisse einer qualitativen Untersuchung, in: TELEVIZION 2, 1999, S. 51 ff.

Howard Sue, Roberts Susan, Moralpanik, in: TELEVIZION 2, 1999, S. $21 \mathrm{ff}$.

Mikos Lothar, Feise Patricia, Herzog Katja, Im Auge der Kamera, Berlin 2000

Lamnek Siegfried, Qualitative Sozialforschung (2 Bände), Weinheim 1993

Lemish Dafna, Tidhar, Chava E., Lebensnahe Mütter, in: TELEVIZION 2, 1999, S. 42 ff.

Moser Heinz, Grundlagen der Praxisforschung, Freiburg 1995

Moser Heinz, Instrumentenkoffer für den Praxisforscher, Freiburg 1998 (2. Aufl.)

Patton Michael Quinn, Qualitative Evaluation and Research Methods, Newbury Park 1990

Richardson Laurel, Evaluating Ethnographie, in Qualitative Inquiry 2000, S. $253 \mathrm{ff}$.

Theunert Helga u.a., Zwischen Vergnügen und Angst - Fernsehen im Alltag von Kindern, Hamburg 1992

Winter Rainer, Der produktive Zuschauer, München 1995

Weinreich Frank, Schulz-Zander Renate, Schulen am Netz - Ergebnisse der bundesweiten Evaluation. Ergebnisse einer Befragung der Computerkoordinatoren und -koordinatorinnen an Schulen, in: Zeitschrift für Erziehungswissenschaft 2000, S. 577 ff. 